\title{
Application de la méthode «Milieu Et Végétaux aquatiques fixés» à 12 rivières françaises : typologie floristique préliminaire
}

\author{
J. Haury 1,2 \\ M. Jaffré 2 \\ A. Dutartre ${ }^{3}$ \\ M.-C. Peltre 4 \\ J. Barbe 5 \\ M. Trémolières 6 \\ M. Guerlesquin ${ }^{7}$ \\ S. Muller ${ }^{4}$
}

Mots clés : rivière, macrophyte, éco-région, méthodologie, typologie.

La végétation de 12 cours d'eau répartis sur le territoire français a été étudiée selon le protocole standardisé d'échantillonnage «Milieu Et Végétaux aquatiques fixés» (M.E.V.) pour tester ce procotole. Le traitement des données par rivière puis pour l'ensemble des séquences étudiées permet d'établir des typologies floristiques. De façon préliminaire, le déterminisme de distribution des phytocénoses est envisagé. Les différences majeures s'expriment à l'amont en fonction de la géologie et de la minéralisation, des conditions d'écoulement et d'ombrage. Une homogénéisation s'effectue vers l'aval, à la faveur de l'eutrophisation et de l'accroissement des dimensions du cours d'eau. La dimension éco-régionale s'exprime d'abord au niveau de la géologie, et secondairement en fonction du climat (atlanticité) et des différences entre rivières de plaine et de piémont. Les résultats sont discutés en vue d'une amélioration du protocole et de la possibilité d'application de la notion de phytocénoses de référence.

Application of the standardized protocol «Milieu Et Végétaux aquatiques fixés» to 12 French rivers : preliminary floristic typology

Keywords : river, macrophyte, eco-region, methodology, typology.

The vegetation of 12 French rivers was studied to test the standardized protocol «Milieu Et Végétaux aquatiques fixés». Data processing for each river and then for all studied stretches gave floristic typologies and an assessment of the pattern of vegetation distribution. A wide diversity appeared upstream, due to geology and mineralization, flow and shade. Vegetation was more homogeneous downstream, following eutrophication and river width increase. The ecoregional features are mainly due to geology, then to distance from the sea and to opposition between foothill and lowland rivers. Results are discussed to improve the methodology, paying attention to reference phytocenosis concept.

1. E.N.S.A. Ecologie et Sciences phytosanitaires, 65 rue de Saint Brieuc, F-35042 Rennes Cedex.

2. I.N.R.A. Ecologie aquatique, 65 rue de Saint Brieuc, F-35042 Rennes Cedex.

3. CEMAGREF Qualité des Eaux, 57, av. de Verdun, Gazinet, F-33612 Cestas.

4. Université de Metz, C.R.E.U.M., 1, rue des Récollets, F-57000 Metz.

5. CEMAGREF Biologie des Ecosystèmes aquatiques, 3bis quai Chauveau, CP220 F-69336 Lyon Cedex 09.

6. Université de Strasbourg, Institut de Botanique, 28 rue Goethe, F-67083 Strasbourg.

7. U.C.O. Institut de Recherches Fondamentales et Appliquées, Biologie végétale, 3 place André Leroy, B.P. 808, F-49008 Angers Cedex 01. 


\section{Introduction}

Les macrophytes sont reconnus comme étant de bons indicateurs de la qualité de l'eau et plus spécialement de son niveau trophique (Kohler 1975, Carbiener et al. 1995). Toutefois, dans le contexte européen de standardisation des méthodes d'évaluation de la qualité de l'eau, la végétation des cours d'eau français est encore mal connue, les études restant très localisées (Haury 1997) : aucune synthèse nationale comparable à celle de Holmes (1983) pour l'Angleterre n'est actuellement disponible.

Pour remédier au manque de connaissances standardisées sur le territoire national, le protocole «Milieu Et Végétaux aquatiques fixés» (M.E.V.) a été élaboré par une équipe pluri-disciplinaire (Université de Metz 1990) et appliqué aux rivières de Lorraine (Grasmück et al. 1993 et 1995). Remanié par le Groupement d'Intérêt Scientifique (G.I.S.) «Macrophytes des eaux continentales», il a été utilisé sur 12 rivières françaises par 6 bureaux d'étude sous l'égide du G.I.S., la standardisation du protocole d'acquisition des données devant faciliter les comparaisons ultérieures. A partir des résultats de chaque rivière, puis de l'ensemble de ces cours d'eau, une typologie préliminaire des phytocénoses des cours d'eau français est recherchée, ainsi qu'une meilleure connaissance de leur déterminisme.

\section{Méthodes et milieux d'étude}

\subsection{Le protocole «Milieu Et Végétaux aquatiques fixés»}

Cette méthode, présentée dans Grasmück et al. (1993), permet un échantillonnage hiérarchisé d'un cours d'eau accessible à pied.

Un découpage abiotique est d'abord réalisé en fonction de la pente, de la géologie, de la largeur, de l'ordre de drainage et des influences anthropiques, ce qui permet de reconnaître des tronçons caractéristiques du fonctionnement morphodynamique du cours d'eau.

Après parcours sur le terrain, des séquences représentatives de l'alternance des faciès et de l'éclairement, d'une longueur minimale de $50 \mathrm{~m}$, sont alors choisies dans le tiers aval de ces tronçons ou dans les principaux affluents.

Les relevés floristiques concernent le lit en eau et la zone supra-aquatique où sont recherchés les macrophytes à partir d'une liste indicative (plus de 300 taxons). Les recouvrements végétaux, exprimés en pourcentages, sont estimés à l'échelle du faciès lors de deux campagnes : en fin de printemps-début été (campagne «printanière») et à l'étiage ou en fin de saison active de végétation (campagne «automnale»), les pé- riodes exactes d'observation dépendant des conditions hydrologiques et climatiques propres aux rivières étudiées.

\subsection{Présentation succincte des 12 rivières}

Les 12 rivières ont été choisies (Fig.1) comme représentatives des éco-régions et des territoires des Agences de l'Eau : l'Aa, l'Aven, la Bresle, le Cousin, la Dronne, le Gapeau, le Guiers, l'Indre, la Lère, la Moder, la Mortagne, la Vinjeanne. Leurs caractéristiques ainsi que les résultats du découpage abiotique sont résumés dans le tableau 1.

\subsection{Traitement des données}

Les données, obtenues au niveau du tronçon (géologie par exemple), de la séquence (éclairement) ou du faciès (recouvrements macrophytiques, granulométrie, profondeur, vitesse de courant), ont d'abord été rapportées aux séquences et harmonisées entre les bureaux d'étude, pour constituer des tableaux homogènes. Des analyses multivariées ont permis d'établir des ensembles floristiques, bases d'une biotypologie des cours d'eau, puis d'étudier les relations entre les facteurs mésologiques et les structures végétales.

Pour chaque rivière, des Analyses en Composantes Principales (A.C.P.) et des Classifications Ascendantes hiérarchiques (C.A.H.) avec le progiciel SPADN (CISIA 1991) ont été faites sur les espèces les plus inféodées à l'eau, algues, bryophytes aquatiques, hydrophytes et hélophytes. Les individus statistiques sont donc des séquences-campagnes; la période ne sera précisée dans le texte que lorsque des différences majeures seront apparues entre les deux campagnes.

Ultérieurement, une A.C.P. a concerné les espèces présentes dans les 539 relevés de la totalité des rivières. Les ensembles floristiques obtenus par C.A.H. déterminent leur biotypologie préliminaire.

Enfin, une Analyse Factorielle Multiple (A.F.M.) (Escofier \& Pagès 1988) a permis d'étudier le déterminisme écologique de distribution des phytocénoses, en prenant en considération les variables du milieu.

Les espèces, avec leurs codes, et les groupes de variables du milieu ou de la végétalisation utilisés dans l'A.F.M., sont inventoriés dans le tableau 2. La nomenclature des genres d'algues suit les flores de Bourrelly (1968, 1970, 1981).

\section{Résultats}

\subsection{Résultats par rivière}

Le nombre de taxons hygrophiles retrouvés par rivière (y compris les formes) varie entre 38 (Gapeau) et 90 (Aa). Pour les taxons strictement aquatiques, il est 


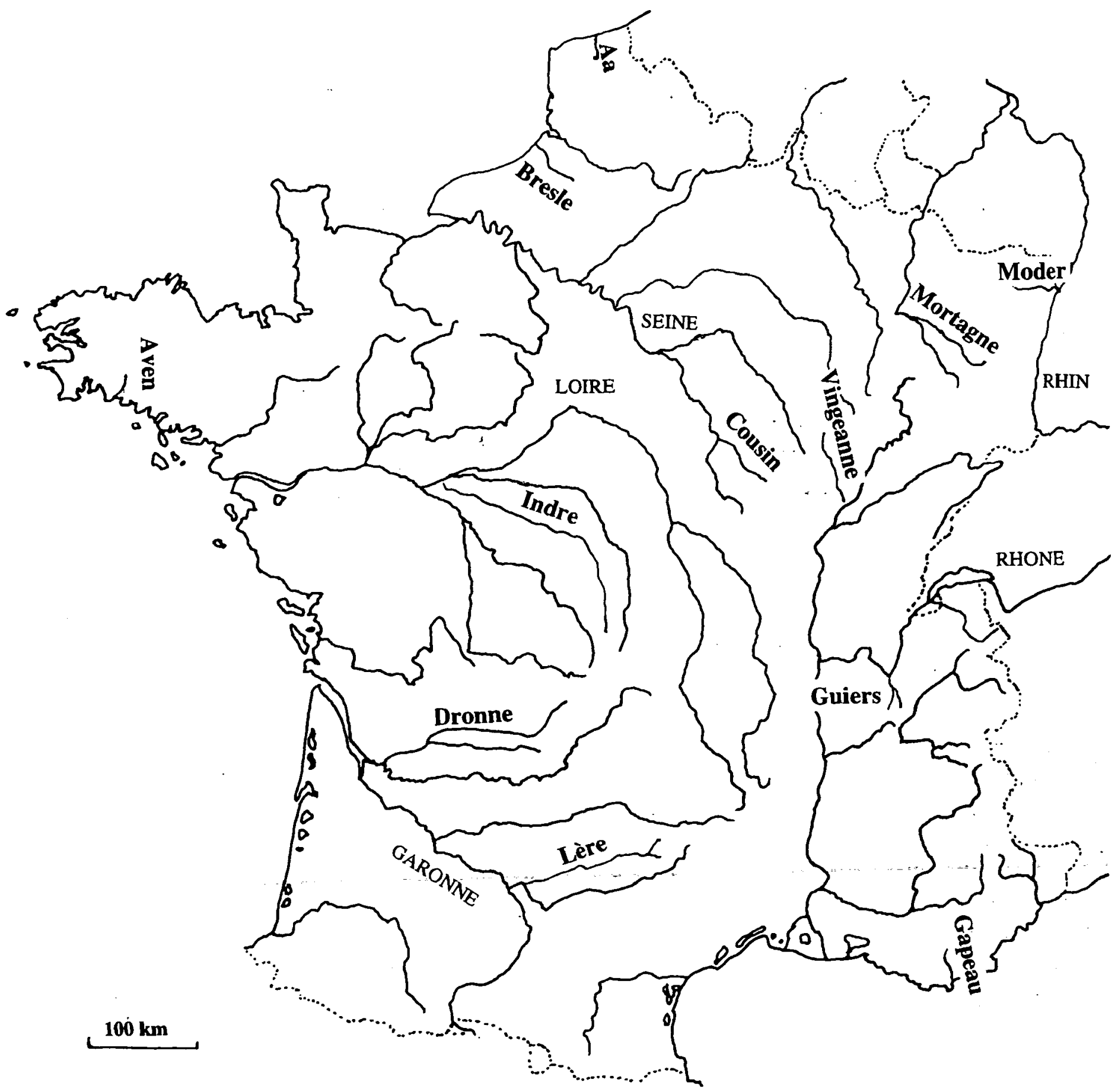

Fig. 1. Localisation des rivières étudiées.

Fig. 1. Location of rivers studied.

plus restreint : 16 exceptionnellement (Guiers où des crues ont réduit fortement la diversité spécifique), 32 (Cousin) à 71 (Moder). Les structures de distribution des phytocénoses sont toujours pluri-dimensionnelles : entre 2 et 8 facteurs significatifs, pour des pourcentages cumulés d'inertie compris entre $29 \%$ et $71 \%$ (Tableau 3).

Dans la majorité des rivières, la prééminence du gradient longitudinal apparaît. Ce dernier est d'autant plus marqué qu'il y a un changement géologique entre l'amont et l'aval (Indre, Mortagne) ou une chenalisation à l'aval (Aa, Indre, Moder); de plus, sur la Moder, une séquence profonde avec des espèces introduites et des lentilles d'eau détermine principalement le facteur 1. Ce gradient est peu net lorsqu'il y a un chaos granitique à l'aval (Aven). Les rivières complexes karstiques (Vingeanne) ou formées de deux bras qui confluent (Guiers Vif et Guiers Mort qui forment le Guiers) dérogent partiellement à ce schéma, avec présence d'espèces potamophiles en cours médian dans une résurgence pour la première et superposition de deux gradients pour le Guiers Vif et le Guiers Mort, le gradient du Guiers aval déterminant le facteur 2. Sur le Cousin, les conditions d'ombrage et d'écoulement as- 
Tableau. 1. Caractéristiques des rivières étudiées.

Table. 1. Characteristics of rivers studied.

\begin{tabular}{|c|c|c|c|c|c|}
\hline Rivières & $\begin{array}{l}\text { Substrats } \\
\text { géologiques } \\
\text { dominants }\end{array}$ & 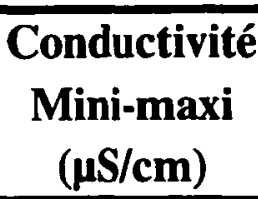 & $\begin{array}{l}\text { Longueur } \\
\text { étudiée } \\
(\mathbf{k m})\end{array}$ & $\begin{array}{c}\text { Nombre de } \\
\text { tronçons } \\
\text { (+ : affluents) }\end{array}$ & $\begin{array}{c}\text { Nombre de } \\
\text { séquences } \\
\text { (+ : affluents) }\end{array}$ \\
\hline$\overline{\mathbf{A a}}$ & Calcaires, marnes & $250-900$ & 56 & 26 & 27 \\
\hline Aven & Granites, grès, schistes & $193-280$ & 33 & 10 & 16 \\
\hline Bresle & Calcaires, craie & $440-530$ & 61 & 17 & 18 \\
\hline Cousin & Granites, gneiss, marne & $40-120$ & 67 & 10 & 13 \\
\hline Dronne & Calcaires, gneiss & $79-412$ & 100 & $20+9$ & $30+9$ \\
\hline Gapeau & Calcaires, r. métamorphiques & $444-844$ & 42 & $10+2$ & $15+4$ \\
\hline Guiers & Calcaires, molasses, sables & $208-530$ & 49 & $17+2$ & $22+3$ \\
\hline Indre & Sables, grès, marnes, calcaires & $79-566$ & 123 & $19+2$ & $29+2$ \\
\hline Lère & Calcaires, molasses & $501-560$ & 34 & $14+6$ & $17+7$ \\
\hline Moder & Grès, marnes & $104-615$ & 90 & 15 & 20 \\
\hline Mortagne & Grès, alluvions & $30-382$ & 75 & $13+1$ & $17+1$ \\
\hline Vingeanne & Calcaires, argiles, grès & $335-508$ & 90 & 11 & 22 \\
\hline
\end{tabular}

sociées à l'homogénéité géologique et à l'importance des peuplements bryophytiques déterminent le premier facteur. Les facteurs secondaires ont des significations écologiques diverses : influence de pisciculture et d'étang (Aven), de pertes puis de résurgences et d'une retenue (Vingeanne), de pollution ponctuelle (Cousin), effets source (Indre) ou affluent (Dronne).

Les ensembles floristiques définis par les classes diffèrent d'une rivière à l'autre, puisque seuls 4 taxons ont été retrouvés en commun : une espèce de bryophyte, Fontinalis antipyretica Hedw. et 3 genres d'algues Vaucheria sp., Cladophora sp. et Oscillatoria sp. Pour chaque rivière, les ensembles floristiques sont néanmoins assez nettement classés. On distingue notamment les groupements fontinaux, bryophytiques calcicoles, potamophiles.

\subsection{Résultats sur l'ensemble des rivières et biotypo- logie générale}

Au total, pour les 324 taxons (ou formes) recensés, 198 noms de la liste indicative n'ont pas été retrouvés, et 74 taxons (ou formes) surtout ripicoles ont été rajoutés.

L'A.C.P. d'ensemble a porté sur 131 espèces les plus inféodées à l'eau. Elle met en évidence une structure penta-dimensionnelle correspondant à $16,6 \%$ d'inertie cumulée, ce qui est beaucoup moins que pour les analyses séparées par rivière. Une forte hétérogénéité ap- paraît, due à quelques séquences de sources $(\mathrm{Aa}$, et dans une moindre mesure Bresle) qui déterminent le facteur 1. L'axe 2 oppose des milieux d'aval canalisés et profonds (Indre, Aa, Moder) à l'ensemble de l'Aven. L'axe 3 oppose les séquences aval de l'Aa, de l'Indre à celles de la Moder (de la deuxième campagne). L'axe 4 oppose l'Aven aux zones amont de la Moder. Le cinquième axe différencie le Guiers et le Gapeau amont de la plupart des séquences de l'Aven. Ces deux derniers axes correspondent à des différences Est-Ouest.

Les C.A.H. définissent des ensembles floristiques. Sur les 27 classes qui ressortent dans le découpage le plus détaillé, 10 ne correspondent qu'à une séquence pour une période : sources de l'Aa, de la Bresle, séquence polluée de la Dronne et séquence aval de l'Aa au printemps, séquences aval du Gapeau et deux séquences aval de la Moder, séquence située à l'aval d'une pisciculture sur l'Aven, et deux séquences amont de la Moder à l'automne. Sur les 17 classes regroupant plusieurs séquences, la classe résiduelle $\left(\mathrm{n}^{\circ} 11: 367\right.$ séquences) est plutôt caractérisée par la présence de Platyhypnidium rusciforme (Neck) Fleisch. et de Ranunculus fluitans Lam.. Dans cette classe se retrouvent des séquences de tous les cours d'eau dont tout le Cousin, l'essentiel de la Lère et de la Dronne. Les autres classes caractérisent une série acidocline (i) et basicline (ii) qui convergent vers des ensembles neutres à basiques eutrophes (iii). 
Tableau 2. Groupes de variables de l'Analyse Factorielle Multiple et liste des macrophytes.

Table. 2. Groups of variables used for the Multiple Factor Analysis, and list of macrophyte taxa.

\begin{tabular}{|c|c|c|c|}
\hline VARIABLES / GROUPES & CODE & VARIABLES / GROUPES & CODE \\
\hline MESOLOGIE PHYSIQUE & & MACROPHYTES (Suite) & \\
\hline 15 variables & & Galium palustre & \\
\hline GEOLOGIE & & Lycopus europaeus & \\
\hline 10 variables & & Mentha aquatica & Mena \\
\hline PHYSICO-CHIMIE & & Myosotis palustris & Myop \\
\hline 3 variables & & Myosotis gr. scorpioides & Myox \\
\hline RECOUVREMENTS VEGETAUX & & Myriophyllum altemiflorum & Myra \\
\hline 10 variables & & Myriophyllum spicatum & Myrs \\
\hline MACROPHYTES & & Myriophyllum verticillatum & \\
\hline Audouinella sp. & Audo & Nasturtium officinale & Naso \\
\hline Batrachospermum sp. & Batr & Nuphar lutea & Nupl \\
\hline Cladophora sp. & Clad & Nymphaea alba & \\
\hline Hydrurus sp. & Hydx & Oenanthe crocata & Oenc \\
\hline Lyngbia sp.+ Phormidium sp. & Lyph & Polygonum amphibium & \\
\hline Melosira sp. & Melo & Polygonum hydropiper & Plgh \\
\hline Rhizoclonium sp. & Rhiz & Ranunculus aquatilis & Rang \\
\hline Spirogyra sp. & Spir & Ranunculus calcareus & Ranc \\
\hline Stigeoclonium sp. & Stig & Ranunculus fluitans & Rann \\
\hline Ulothrix sp. & Ulot & Ranunculus peltatus & Ranl \\
\hline Vaucheria sp. & Vauc & Ranunculus penicillatus & Ranp \\
\hline Chiloscyphus polyanthus & Chip & Ranunculus trichophyllus & \\
\hline Porella pinnata & Porp & Rorippa amphibia & \\
\hline Scapania undulata & Scau & Veronica anagallis aquatica & Vera \\
\hline Hygroamblystegium fluviatile & Hygf & Veronica beccabunga & \\
\hline Leptodictyum riparium & Lepr & Agrostis stolonifera & Agrs \\
\hline Hygroamblystegium tenax & Hygt & Alisma plantago-aquatica & Alip \\
\hline Brachythecium rivulare & Brav & Butomus umbellatus & Butu \\
\hline Cinclidotus aquaticus & Cina & Elodea canadensis & Eloc \\
\hline Cinclidotus fontinaloides & Cinf & Egeria densa & \\
\hline Cratoneurum filicinum & Craf & Elodea nuttallii & Elon \\
\hline Fissidens crassipes & Fisc & Glyceria fluitans & \\
\hline $\begin{array}{l}\text { Fissidens pusillus } \\
\text { Fissidens rivularis }\end{array}$ & Fisp & $\begin{array}{l}\text { Glyceria maxima } \\
\text { Groenlandia densa }\end{array}$ & Glym \\
\hline Fontinalis antipyretica & Fona & Hydrocharis morsus-ranae & \\
\hline Fontinalis durieui & Fond & Iris pseudacorus & \\
\hline Fontinalis squamosa & & Lemna gibba & Lemg \\
\hline Hyocomium flagellare & & Lemna minuscula & Lemi \\
\hline Hygrohypnum ochraceum & Hygo & Lemna minor & Lemm \\
\hline Octodiceras fontanum & Octf & Lemna trisulca & Lemt \\
\hline Platyhypnidium rusciforme & Oxyr & Phalaris arundinacea & Phaa \\
\hline Apium inundatum & Heli & Phragmites australis & \\
\hline Apium nodiflorum & Heln & Potamogeton crispus & Potr \\
\hline Berula erecta & Bere & Potamogeton nodosus & Potd \\
\hline Callitriche hamulata & Calh & Potamogeton pectinatus & Pots \\
\hline Callitriche palustris & Calp & Potamogeton perfoliatus & \\
\hline Callitriche platycarpa & Caly & Sagittaria sagittifolia & Sags \\
\hline Callitriche stagnalis & Cals & Scirpus lacustris & Scil \\
\hline Callitriche truncata & & Sparganium emersum & \\
\hline Caltha palustris & Cltp & Sparganium erectum & \\
\hline Cardamine amara & & Sparganium minimum & Spam \\
\hline Ceratophyllum deme & Cerd & $\begin{array}{l}\text { Spirodela polyrhiza } \\
\text { Zannichellia palustris }\end{array}$ & Lemp \\
\hline
\end{tabular}


Tableau 3. Résultats des Analyses en Composantes Principales par rivière.

Table 3. Results of Principal Component Analyses on each river.

\begin{tabular}{lccll}
\hline Rivières & $\begin{array}{l}\text { Nombre NB } \\
\text { (NB) de }\end{array}$ & $\begin{array}{l}\text { Inertie } \\
\text { cumulée } \\
\text { Taxons }\end{array}$ & $\begin{array}{l}\text { Signification } \\
\text { axes }\end{array}$ & $\begin{array}{l}\text { écologique } \\
\text { des axes }\end{array}$ \\
\hline Aa & 90 & $3(5)$ & $28,8(39,9)$ & Gradient longitudinal (gradient), effet source \\
Aven & 61 & 5 & 49,4 & Gradient + trophie, profondeur, éclairement, gorges, pollution \\
Bresle & 60 & 5 & 45,1 & Gradient, eutrophisation \\
Cousin & 41 & $4(6)$ & $54,6(70,8)$ & Eclairés-lents/ombragés-rapides, qualité des eaux, gradient \\
Dronne & 54 & 5 & 33,8 & Gradient, effet affluent, vitesse/profondeur \\
Gapeau & 38 & 5 & 52,5 & Prélèvement d'eau, pollution, géologie, effet affluent \\
Guiers & 70 & 4 & 45,7 & Gradient + effet affluent, éclairement, végétalisation \\
Indre & 74 & $3(8)$ & $28,9(53,0)$ & Gradient, effet source, partie chenalisée aval \\
Lère & 50 & $3(5)$ & $36,2(49,1)$ & Gradient, effet milieu karstique \\
Moder & 71 & $3(6)$ & $33,2(51,4)$ & Gradient + profondeur, adventices, hélophytes, courant \\
Mortagne & 58 & $4(6)$ & $46,7(62,4)$ & Gradient, pollution \\
Vingeanne & 57 & 2 & 29,0 & Gradient, assec/débit soutenu \\
\hline
\end{tabular}

(i) Typique des sources oligotrophes acides, une séquence amont de $l^{\prime}$ Aven ( $n^{\circ} 27: 2$ dates) est caractérisée (par ordre décroissant d'inféodation significative à la classe) par Juncus bulbosus L., Scirpus fluitans L., Glyceria fluitans (L.) R. Br., Batrachospermum sp., Scapania undulata (L.) Dum., Potamogeton polygonifolius Pourret, Fissidens pusillus Wils., Polygonum amphibium L., Ulothrix sp.. Les sources de la Moder $\left(\mathrm{n}^{\circ} 22: 2\right.$ séquences printanières) sont caractérisées par Chrysosplenium oppositifolium L., Chrysosplenium alternifolium. L. et Equisetum fluviatile. L.. Des séquences amont acides de l'Aven et un affluent de la Dronne ( $\mathrm{n}^{\circ} 16: 5$ séquences) sont caractérisés par $\mathrm{Cal}$ litriche stagnalis Scop., Callitriche hamulata Kütz ex Koch, Hyocomium flagellare (Dicks.) B. e., Oenanthe crocata L., Cardamine amara L., Leptodictyum riparium (Hedw.) Warnst., $G$. fluitans, $F$. antipyretica, $P$. amphibium. L'essentiel de l'Aven ( ${ }^{\circ} 15: 18$ séquences) est colonisé par Fontinalis squamosa Hedw, O. crocata, Octodiceras fontanum (La Pyl.) Lindb., Ranunculus penicillatus (Dum.) Bab., Porella pinnata Lindb., Myriophyllum alterniflorum DC., Chiloscyphus polyanthus (L.) Corda, C. hamulata, L. riparium, Riccardia sinuata (Dicks) Trev., F. pusillus, Brachythecium rivulare B. e., Potamogeton gramineus L., Apium inundatum (L) Reichenb. fil., Potamogeton panormitanus Biv.. La Moder médiane ( ${ }^{\circ} 19: 3$ séquences printanières) est eutrophisée, avec Ranunculus peltatus Schrank, Callitriche obtusangula Le Gall, Elodea ernstiae $\mathrm{S}^{\mathrm{T}} \mathrm{John}, C$. hamulata ; ses peuple- ments automnaux diffèrent ( $\mathrm{n}^{\circ} 20: 3$ séquences) avec Callitriche palustris L., Elodea canadensis Michx , $R$. penicillatus, $C$. hamulata.

(ii) L'essentiel du Guiers et 3 séquences du Gapeau ( ${ }^{\circ} 14: 28$ séquences) correspondent aux rivières calcaires de piémont, dominées par des bryophytes et des algues : Cinclidotus aquaticus (Jaeg.) B. e., Cinclidotus fontinaloides (Hedw.) P. Beauv., B. rivulare, Cratoneurum filicinum (Hedw.) Roth, Hydrurus sp., Melosira sp., Fontinalis durieui Schimp., Ulothrix sp., Fissidens rivularis (Spruce) B. e., Stigeoclonium sp.. Les ruisseaux calcaires plutôt lents de l'Aa (n ${ }^{\circ} 17: 5$ séquences) sont caractérisés par Ranunculus aquatilis L., Ranunculus circinatus Sibth., Philonotis tomentella Moll., Wolffia arrhiza (L.) Horkel ex Wimmer, Vaucheria sp., Phalaris arundinacea L. et Myosotis palustris Roth. Les ruisseaux plutôt eutrophisés de la Lère, de l'Aa, de la Bresle, de l'Indre, de l'essentiel du Gapeau ( $n^{\circ} 12: 45$ séquences) sont caractérisés par $\mathrm{Cla}$ dophora sp., Scirpus lacustris L., Mentha aquatica L., Veronica beccabunga L., Alisma plantago-aquatica L., Fissidens crassipes Wils, Berula erecta (Hudson) Coville, Glyceria maxima (Hartman) Holmberg, Ranunculus omiophyllus Ten, Callitriche truncata Guss., Sparganium erectum L., Agrostis stolonifera L., . Melosira sp., Potamogeton obtusifolius Mert. \& Koch, Spirogyra sp., Elodea nuttallii (Planchon) $\mathrm{S}^{\mathrm{T}} \mathrm{John}, \mathrm{Vau}-$ cheria sp.. Un secteur du Gapeau ( $\mathrm{n}^{\circ} 24: 1$ séquence, 2 dates) est caractérisé par Alisma lanceolata With., Lycopus europaeus L., Groenlandia densa Fourr., Me- 
losira sp., Mentha aquatica L., Veronica anagallisaquatica L., Spargnium erectum; Spirogyra sp.. Un affluent de la Dronne ( $n^{\circ} 23: 1$ séquence, 2 dates) possède un groupement original à Potamogeton friesii Rupr., Potamogeton gramineus L., C. polyanthus, Apium nodiflorum (L.) Lag., Chara sp. et Caltha palustris L.. Les phytocénoses planitiaires calcicoles eutrophisées de l'Aa et surtout de la Bresle ( $\mathrm{n}^{\circ} 13: 36$ séquences) sont constituées par : Zannichellia palustris L., V. anagallis-aquatica, M. palustris, B. erecta, Callitriche platycarpa Kütz, E. canadensis, Nasturtium officinale R. Br., Vaucheria sp., Phalaris arundinacea, A. stolonifera, Potamogeton crispus L., C. obtusangula, Myosotis gr. scorpioides., L. riparium, G. densa, Galium palustre L. et Lemna minor L..

(iii) L'aval de l'Indre ( ${ }^{\circ} 18: 4$ séquences) est caractérisé par Rorippa amphibia (L.) Besser, Myriophyllum spicatum L., S. lacustris, Phragmites australis (Cav.) Trin. ex Steudel (L.) Sibth. \& Sm., Nuphar lutea (L.) Sibth. et Sm., P. amphibium, Sparganium emersum L., Lemna minuscula Herter, Myosotis gr. scorpioides L., Ceratophyllum demersum L.. L'aval de l'Indre et une séquence de la Moder ( $\mathrm{n}^{\circ} 23: 3$ séquences) sont des secteurs lents et eutrophisés caractérisés par L. minuscula, Spirodela polyrhiza (L.) Schleiden, N. lutea, Butomus umbellatus L., Potamogeton pectinatus L., C. demersum, Sagittaria sagittifolia L. et Potamogeton nodosus Poiret. Le secteur le plus aval et canalisé de l'Aa ( $\mathrm{n}^{\circ} 25: 1$ séquence, 2 dates) possède une végétation assez comparable avec Acorus calamus L., P. australis, S. sagittifolia, S. polyrhiza, C. demersum, $N$. lutea, $S$. erectum, L. minor. Deux séquences printanières de l'aval de l'Aa $\left(\mathrm{n}^{\circ} 26\right)$ sont assez proches, avec Potamogeton lucens L., Hydrocharis morsus-ranae L., Potamogeton trichoides Cham. \& Schrader, S. sagittifolia, C. demersum, S. polyrhiza, $N$. lutea.

L'A.F.M. est réalisée sur 130 variables réparties en 5 groupes. Ses résultats généraux sont présentés dans le tableau 4. La structure est également penta-dimensionnelle, cumulant $25,0 \%$ de l'inertie totale. Le groupe de la mésologie (15 variables) est assez mal représenté sur le premier facteur commun (seulement $59 \%$ ), mais beaucoup mieux sur les 4 suivants; il est dominant pour le second facteur. Le groupe de la géologie (10 variables) est surtout bien représenté sur les deux premiers facteurs. Celui de la qualité de l'eau (3 variables) participe essentiellement au premier facteur et peu au second. Les caractéristiques de la végétalisation (10 variables) sont bien représentées sur les 4 premiers facteurs. Les recouvrements macrophytiques des espèces les plus fréquentes (92 variables) déterminent largement la structure des 5 premiers facteurs. L'examen du premier plan factoriel des variables (Figure 2) met en évidence un premier axe lié à la végétalisation, la conductivité et donc les substrats calcaires qui s'opposent au fond nu, aux granites et grès. Les ensembles floristiques qui ressortent sont alors les calcicoles ou espèces eutrophes (B. erecta, Vaucheria sp., Cladophora sp., A. stolonifera, M. palustris, V. anagallisaquatica, $N$. officinale, $C$. platycarpa, $P h$. arundina$c e a)$ qui se différencient de quelques acidoclines $(C$. polyanthus, $S$. undulata, $P$. pinnata, $F$. pusillus). Sur le deuxième axe, les substrats fins, les roches érodables et perméables ainsi que l'éclairement, la profondeur et la largeur, associés à de forts recouvrements d'hydrophytes nageants ou flottants, s'opposent aux substrats grossiers soumis à de fortes vitesses de courant et co-

Tableau 4. Principaux résultats généraux de l'Analyse Factorielle Multiple.

Table 4. Main statistical results of the Multiple Factor Analysis.

\begin{tabular}{|c|c|c|c|c|c|}
\hline Paramètres $\mathbb{N}^{\circ}$ des facteurs & 1 & 2 & 3 & 4 & 5 \\
\hline \multicolumn{6}{|l|}{ Analyse générale } \\
\hline Valeurs propres de l'analyse générale & 2,34 & 1,82 & 1,50 & 1,30 & 1,05 \\
\hline Pourcentages d'inertie & 7,30 & 5,66 & 4,66 & 4,06 & 3,26 \\
\hline \multicolumn{6}{|c|}{ Corrélations entre les variables des groupes et les variables de l'analyse générale } \\
\hline Groupe mésologie & 0,590 & 0,836 & 0,684 & 0,661 & 0,755 \\
\hline Groupe géologie & 0,768 & 0,648 & 0,496 & 0,534 & 0,472 \\
\hline Groupe qualité de l'eau & 0,775 & 0,320 & 0,429 & 0,570 & 0,393 \\
\hline Groupe recouvrements végétaux & 0,733 & 0,645 & 0,557 & 0,599 & 0,441 \\
\hline Groupe macrophytes & 0,771 & 0,813 & 0,821 & 0,805 & 0,756 \\
\hline \multicolumn{6}{|c|}{ Rapport inertie inter-groupes/inertie totale } \\
\hline & 0,529 & 0,443 & 0,370 & 0,405 & 0,329 \\
\hline
\end{tabular}


lonisés par les bryophytes : c'est donc partiellement un axe de gradient longitudinal. Les groupements qui en résultent sont les potamophiles (C. demersum, $N$. lutea,...$)$ dominés par les spermaphytes qui s'opposent aux bryophytes plutôt calcicoles ( $C$. aquaticus, $C$. filicinum) ou indifférents (B. rivulare, $C$. fontinaloides). Le troisième axe correspond à une opposition entre les séquences profondes sur argiles au pH élevé, et les milieux courants sur granites, caractérisés notamment par $O$. crocata, $R$. penicillatus, C. platycarpa, C. hamula- ta, voire $V$. anagallis-aquatica. Le quatrième axe correspond au gradient de température, l'augmentation de ce paramètre.s'opposant aux grès et à l'acidité des roches : il traduit la structure géologique fréquente de roches acides à l'amont, et basiques à l'aval. Le cinquième axe est essentiellement déterminé par la granulométrie grossière et les granites, les blocs et rochers étant colonisés par les bryophytes. Au niveau des individus, les résultats concordent avec la typologie établie avec l'A.C.P., avec un moindre poids des séquences à

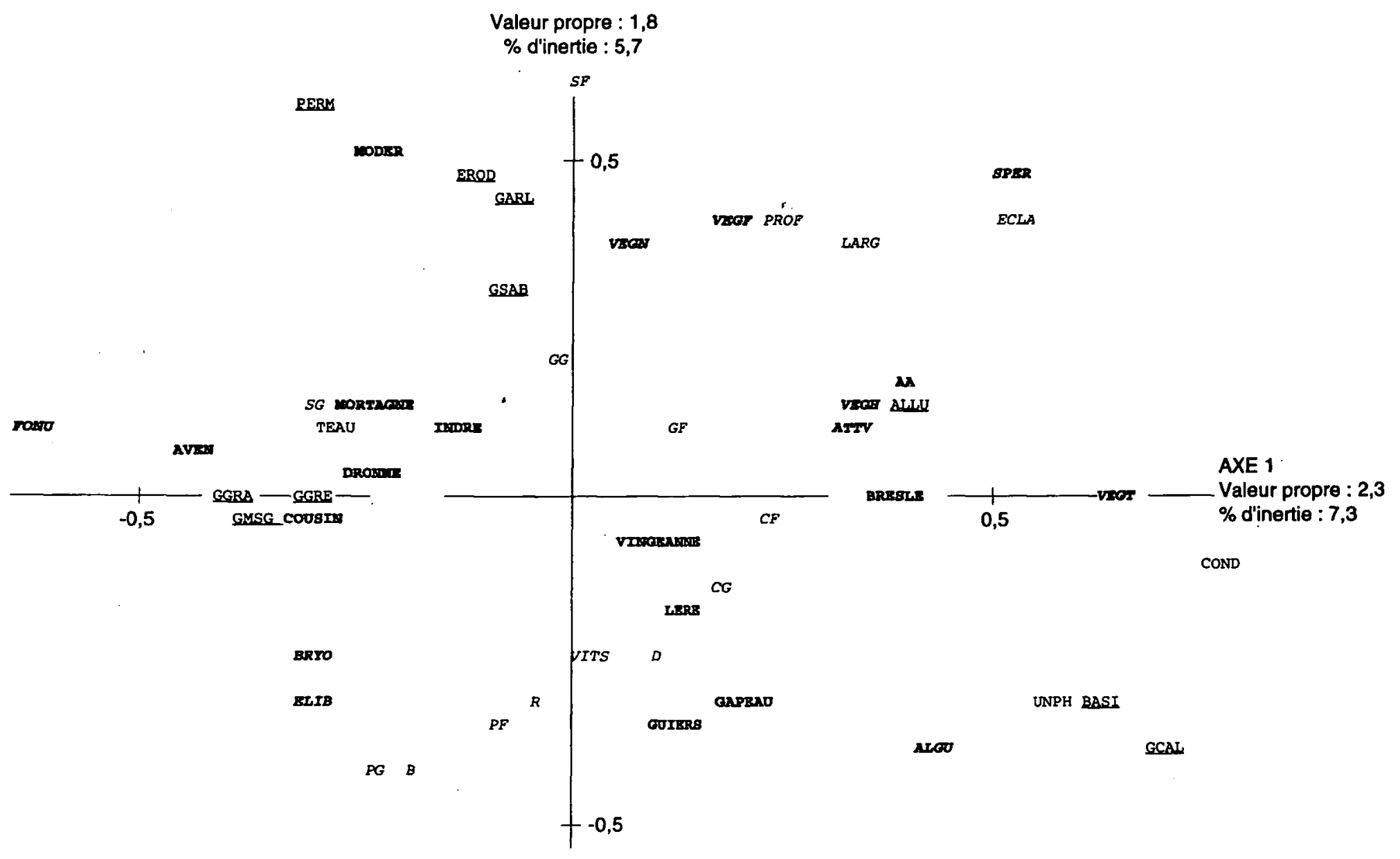

Fig. 2. Plan factoriel F1 x F2 de l'A.F.M. (539 séquences, 130 variables réparties en 5 groupes) et projection des rivières. a. Caractéristiques mésologiques, géologiques, physico-chimiques et de végétalisation - Projection des rivières.

Légende : MESOLOGIE - LARG : Largeur; PROF : Profondeur ; VITS : Vitesse ; ECLA : Eclairement ; SF : Sables fins ; SG : Sables grossiers ; $G F$ : Graviers fins ; $G G$ : Graviers grossiers ; $C F$ : Cailloux fins ; $C G$ : Cailloux grossiers ; $P F$ : Pierres fines ; $P G$ : Pierres grossières ; $B$ : Blocs ; $R$ : Rochers ; $D$ : Dalles. GEOLOGIE - GCAL : Calcaires et marnes ; GSAB : Sables ; GARL : Argiles et limons ; GGRE : Grès ; GM$S G$ : Micaschistes, schistes et gneiss ; GGRA : Granites ; ALLU : Alluvions ; BASI : Caractère basique des roches ; PERM : Perméabilité ; EROD : Erodabilité. PHYSICO-CHIMIE - TEAU : Température de l'eau ; UNPH : pH ; COND : Conductivité. RECOUVREMENTS VEGETAUX - ELIB : \% Eau libre ; FONU : \% Sol nu ; VEGT : Végétation totale ; ALGU : \% Algues ; BRYO : \% Bryophytes ; SPER : \% Hydrophytes ; VEGN : \% Végétation nageante ; VEGF : \% Végétation flottante ; VEGH : \% Végétation hélophytique ; ATTV : \% Atterrissement végétal. RIVIERES : les rivières sont présentées en caractères majuscules gras.

Fig. 2. Factorial Plane F1xF2 of Multiple Factor Analysis ( 539 individuals, 130 variables into 5 groups) and projection of rivers. a. Mesology, geology, physico-chemistry and general plant cover - Projection of rivers.

Legend : PHYSICAL FEATURES - LARG : Width ; PROF : Depth ; VITS : Current velocity ; ECLA : clarity ; $S F$ : fine sand ; SG : Coarse sand ; $G F$ : fine gravel ; $G G$ : Coarse gravel ; $C F$ : fine pebbles ; $C G$ : Coarse pebbles ; $P F$ : small stones ; $P G$ : Coarse stones ; $B:$ Blocks ; $R:$ Boulders ; $D$ : Flatstones. GEOLOGY - GCAL : Chalk and limestones ; GSAB : Sands ; GARL : Clay and silt ; GGRE : Sandstones ; GMSG : Schist and gneiss ; GGRA : Granites ; ALLU : Alluvium ; BASI : Basic stones ; PERM : Permeability ; EROD : Erodeability. PHYSICO-CHEMISTRY - TEAU : Water temperature ; UNPH : $\mathrm{pH}$; COND : Conductivity. PLANT COVER - ELIB : \% Bare water ; FONU : \% Bare substratum ; VEGT : Total cover ; ALGU : \% Algae ; BRYO : \% Bryophyta ; SPER : \% Hydrophytes ; VEGN : \% Underwater vegetation ; VEGF : \% Floating vegetation ; VEGH : \% Emergent vegetation ; ATTV : \% Covered silt. RIVERS are indicated with bold capital letters. 


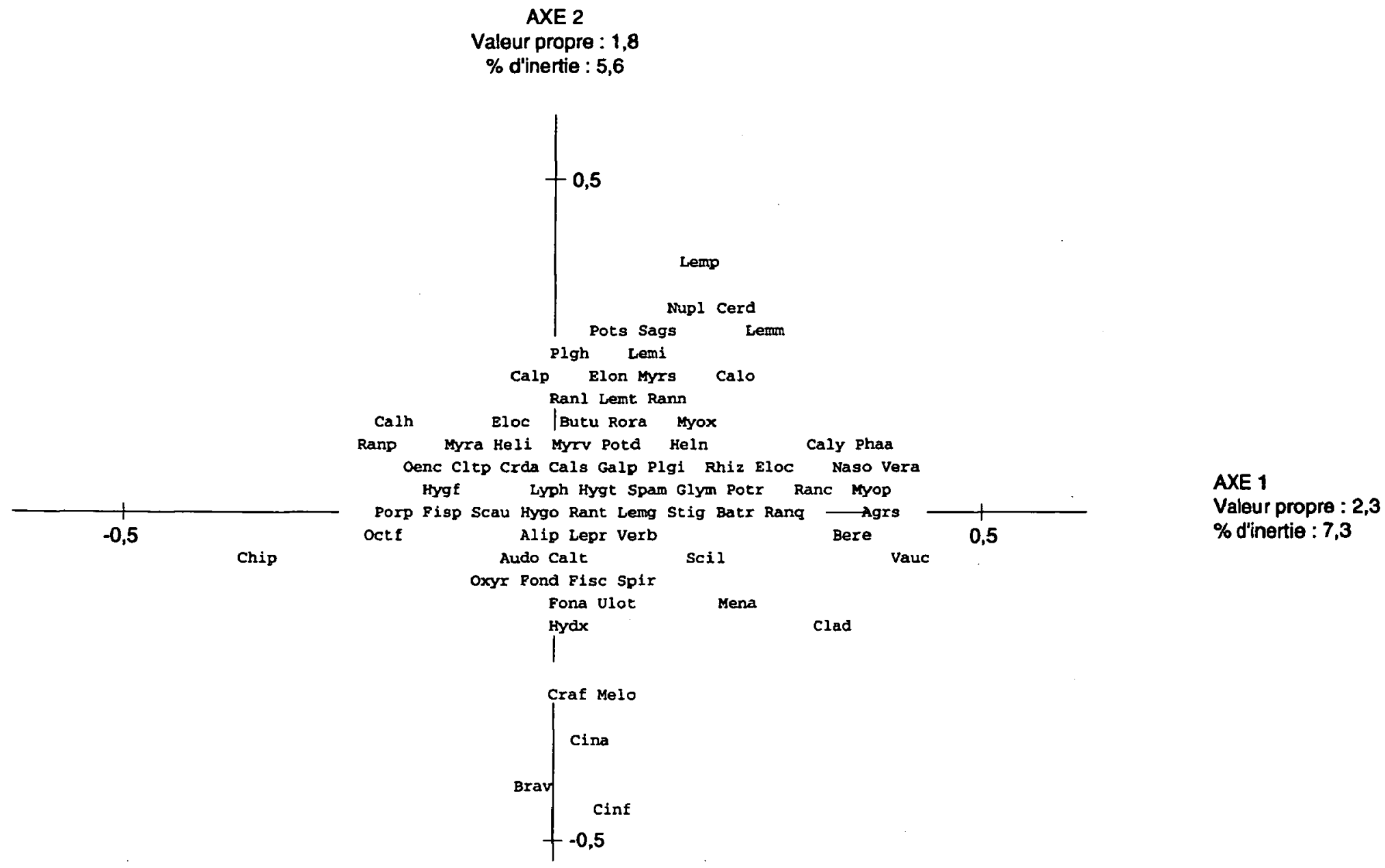

Fig. 2. Plan factoriel F1 x F2 de l'A.F.M. (539 séquences, 130 variables réparties en 5 groupes) et projection des rivières. b. Macrophytes.

Légende : Seules les espèces les plus éloignées de l'origine sont présentées. Pour la signification des sigles, voir le tableau 2.

Fig. 2. Factorial Plane F1xF2 of Multiple Factor Analysis (539 individuals, 130 variables into 5 groups) and projection of rivers.

b. Macrophytes.

Legend : Only the variables far from the centre are listed. For the significance of abbreviations, see table 2.

flore rare, en raison du tri effectué. Le premier facteur correspond aux cours d'eau calcaires côtiers, Aa et Bresle. Le second facteur est déterminé par les séquences aval profondes canalisées de la Moder et de l'Indre qui s'opposent au Guiers, à l'amont de la Lère et du Gapeau. Le troisième facteur discrimine les séquences aval de l'Aa de celles de sa source. Le quatrième facteur est associé aux secteurs lents et profonds de la Moder, de l'Indre et du Gapeau. Le cinquième facteur est principalement déterminé par l'Aven. La répartition des rivières dans le premier plan factoriel montre les différences «éco-régionales».

\section{Discussion}

\subsection{Apports et limites de ce jeu de données}

Malgré la non-exhaustivité taxonomique de ce jeu de données, la couverture importante du territoire national qu'il représente permet de conforter les renseignements acquis plus localement, tout en enrichissant la liste indicative des macrophytes des cours d'eau français d'une douzaine de taxons (notamment des espèces introduites ou considérées comme exclusivement stagnophiles). La sous-estimation de la richesse floristique réelle des rivières par cette méthode résulte d'une distribution localisée de beaucoup d'espèces, et d'une longueur relativement restreinte des relevés. Ainsi, sur le Scorff (Bretagne-Sud), la perte taxonomique de la méthode M.E.V. par rapport à un échantillonnage plus important sur un même tronçon (mis en oeuvre pour un objectif d'exhaustivité) atteint $130 \%$ (Haury 1996). Le parcours de linéaires plus longs (voire de la totalité des rivières - méthode de Kohler 1975, avec des inventaires localisés plus précis - Pr G. Janauer, Univ. Vienne, comm. pers.) est préconisé par Holmes (1983) et par Carbiener et al. (1995). Toutefois, la recherche de séquences représentatives, postulat du protocole MEV destiné à obtenir une vision réaliste de la végétalisation et de la qualité des cours d'eau, amène à ne pas recenser systématiquement les phytocénoses les plus diver- 
sifiées et à taux de végétalisation maximal qui sont $a$ priori les plus proches des phytocénoses de référence. Enfin, une autre limite concerne la taxonomie, certains macrophytes de petite taille ou de détermination délicate (renoncules, callitriches, bryophytes) ayant pu être confondus ou omis.

\subsection{Végétation des rivières et approche du détermi- nisme des phytocénoses}

Pour ce que l'on connaît de la végétation des rivières dans les différentes éco-régions, les résultats M.E.V. sont globalement en accord avec la bibliographie : Thoen et al. (1996) pour la Bresle et l'Aa, Haury (1996) pour l'Aven, Verneaux (1973), Trémolières et al. (1994), Grasmück et al. (1995), Thiébaut et Muller (1995) et Robach et al. (1996) pour les rivières du Nord-Est de la France. Différentes références assez éloignées permettent des comparaisons avec certaines rivières comme l'étude des rivières de Lozère (Codhant et al. 1991) pour le Guiers. En revanche, pour l'Indre, le Gapeau, la Lère, la Dronne, les références bibliographiques manquent.

Les biocénotypes ressortent bien par l'analyse séparée des rivières, mais sont partiellement «gommés» par le mélange de l'ensemble des rivières, contrairement à ce qui est ressorti en Lorraine (Grasmück et al. 1995). Ceci est dû au nombre important de séquences particulières, pourtant choisies comme représentatives des tronçons. La faible intensité d'échantillonnage (au plus 2 séquences étudiées par tronçon) ne permet alors pas d'avoir suffisamment de relevés pour essayer de cerner le déterminisme de leurs phytocénoses. Les différences saisonnières sont notables pour certaines rivières (Moder) et justifient l'étude à deux périodes.

On retrouve les groupes mis en évidence par Haslam (1987) en fonction de la trophie, notamment pour les classes oligotrophes et hypertrophes, mais, compte-tenu de l'importance des bryophytes dans la définition des typologies floristiques, il s'avère indispensable d'affiner sa démarche. De même, certains groupements sont très proches de ceux décrits par Holmes (1983), ce qui avait déjà permis des comparaisons écorégionales pour le Callitrichetum hamulatae (Haury et al. 1995).

A l'échelle de la France, le poids des facteurs géologiques et des gradients biogéographiques, souvent sous-estimés dans les études localisées, ressort pleinement. Le «fond floristique commun» correspond à des végétations moyennement rhéophiles développées sur des substrats très variés : cette convergence entre les séries de différents substrats géologiques corrobore les observations de Grasmück et al. (1995) et de Robach et al. (1996), mais sur un territoire beaucoup plus vaste. Les facteurs hydrodynamiques locaux déterminent, d'une part, une sélection drastique de macrophytes en rivières de piémont, et, d'autre part, des végétations potamophiles qui sont souvent très eutrophes dans les zones alluviales, ce qui inciterait, à l'instar de Bornette \& Amoros (1991) et de Eglin \& Robach (1992), à $s$ 'intéresser aux relations entre hydrologie quantitative et chimie de l'eau pour en comprendre le déterminisme. La végétation calcicole apparaît comme plus variée à l'aval, mais c'est probablement un artefact de l'échantillon, l'aval des grands cours d'eau étant le plus souvent calcaire, ce qui avait posé des problèmes similaires d'interprétation à Grasmück et al. (1995). Le milieu physique se traduit par le gradient longitudinal, mais aussi par l'hydrodynamique locale (résurgences et pertes, chenalisation, anse profonde). Dans cette étude, la minéralisation (résultante de la géologie) et l'eutrophisation (souvent associée au gradient longitudinal, mais aussi due à des pollutions ponctuelles) semblent difficiles à dissocier dans les zones aval. L'originalité éco-régionale des systèmes atlantiques acides (Aven) ou calcaires (Aa et Bresle) ressort, ainsi que celle des rivières de piémont (Guiers). Les parts respectives de l'hydrodynamique - et des stratégies de colonisation des macrophytes qu'elle sélectionne - et celles des paramètres de la qualité de l'eau restent donc à déterminer.

\section{Conclusion}

Ce travail de traitement des données met en évidence la difficulté d'échantillonner les rivières pour obtenir une vision «représentative» de leurs phytocénoses: beaucoup de cas particuliers ressortent sans que leur déterminisme puisse être pleinement élucidé. La nécessité de conforter l'échantillon en ayant plusieurs rivières par éco-région ressort pour mieux mettre en évidence les caractères généraux des phytocénoses des cours d'eau français et avoir des réplicats pour ces cas particuliers.

Toutefois, l'accroissement des connaissances est important sur des rivières où aucune donnée floristique n'était disponible. La disparité des listes entre les résultats d'une recherche détaillée et celles obtenues lors de cette mise en oeuvre du protocole M.E.V. permet de jauger l'écart existant entre un inventaire simple (visant à qualifier rapidement la diversité et l'état «moyen» de la végétation des cours d'eau) et des «phytocénoses de référence» traduisant la végétation «potentielle» (pouvant coloniser le milieu physique en absence de perturbations des biotopes et de la qualité physico-chimique des eaux). Ces différences entre les 
phytocénoses observées et les végétations «optimales» (peuplements macrophytiques les plus diversifiés ou les plus recouvrants sur les tronçons étudiés) devraient permettre d'établir un diagnostic plus précis de la qualité des milieux anthropisés que sont les rivières.

Ce protocole doit donc continuer à être testé sur d'autres rivières, y compris en milieu montagnard, méditerranéen et dans les marais, avec quelques améliorations : allongement des séquences, maintien d'un parcours minimal pour les choisir, vérifications taxonomiques par des experts, réalisation d'analyses d'eau. Les données obtenues, intégrées dans une base de données, devraient contribuer à cette connaissance minimale du «compartiment» macrophytes pour qualifier l' «état de santé des hydrosystèmes» recherchée dans les Systèmes d'Evaluation de la Qualité des Agences de l'Eau. Les informations apportées par les macrophytes pourraient alors être comparées à celles des compartiments autres retenus dans cette démarche, c'est-à-dire les diatomées, les invertébrés et les poissons.

\section{Remerciements}

Les auteurs remercient les 6 Agences de l'Eau qui ont financé l'ensemble du programme et leur ont accordé l'autorisation d'utiliser les résultats pour leur valorisation scientifique, ainsi que les 6 bureaux d'étude qui ont acquis les données présentées : Association des Médecins et Biologistes pour l'Environnement (A.M.B.E.Valenciennes : J.-L. Mériaux), AQUASCOP (Nancy et Angers : N. Grasmück, K. Pobis), Cellule d'Application en Ecologie (C.A.E.Dijon : C. Chauvin), Phytolab (Dampierre - 39 : G. Bailly), Groupe d'Etudes et de Recherches en Ecologie Appliquée (G.E.R.E.A.Martignac - 33 : J. Dulong), Société d'Ingénierie pour l'Eau et l'Environnement (S.I.E.E.-Montpellier : M. Braud).

Les auteurs (M. Jaffré excepté) participent au Groupement d'Intérêt Scientifique (G.I.S.) : «Macrophytes des eaux continentales».

\section{Travaux cités}

Bornette G. \& Amoros C. 1991. - Aquatic vegetation and hydrology of a braided river floodplain. J. Veg. Sci. $2: 497-512$.

Bourrelly P. 1968, 1970, 1981. - Les algues d'eau douce. I - Algues vertes, II - Algues jaunes et brunes (2ème éd.), III - Algues bleues et rouges. Boubée, Paris : 511 p., 517 p., 512 p.

Carbiener R., Trémolières M. \& Muller S. 1995. — Végétation des eaux courantes et qualité des eaux : une thèse, des débats, des perspectives. Acta bot. Gallica, 142 (6) : 489-531.

CISIA (Centre International de Statistique et d'Informatique Appliquées) 1991. - SPADN2.0 intégré. Saint Mandé, France.

Codhant H., Valkman G., Haury J. \& Dutartre A. 1991. — Les macrophytes aquatiques bioindicateurs de la qualité des eaux courantes - Département de la Lozère. Rapp. int. Contr. CEMAGREF-I.N.R.A., Conseil Général. CEMAGREF Bordeaux, I.N.R.A. Rennes, Conseil Général de Lozère : 146 p.
Eglin I. \& Robach F., 1992. — Typologie et végétation de l'hydrosystème rhénan dans le secteur central de la plaine d'Alsace : interprétation et fonctionnement écologique. Thèse de Doctorat, Université Louis Pasteur, Strasbourg : 342 p.

Escofier B. \& Pagès J. 1988. — Analyses factorielles simples et multiples - Objectifs, méthodes et interprétation. Dunod éd. Paris : $241 \mathrm{p}$.

Grasmück N., Haury J., Léglize L. \& Muller S. 1993. — Analyse de la végétation aquatique fixée des cours d'eau lorrains en relation avec les paramètres d'environnement. Annls. Limnol., 29 (3-4) : 223-237.

Grasmück N., Haury J., Léglize L. \& Muller S. 1995. — Assessment of the bioindicator capacity of aquatic macrophytes using multivariate analysis. Hydrobiologia, 300-301 : 115-122.

Haslam, S.M., 1987. - River plants of Western Europe. Cambridge Univ. Press, Cambridge : $512 \mathrm{p}$.

Haury J. 1996.. - Macrophytes des cours d'eau : bioindication et habitat piscicole. Thèse d'Habilitation à Diriger des Recherches, Université de Rennes I. 3 vol. : 99 p. +2 vol. n.p.

Haury J. 1997. - Les macrophytes, estimateurs de la qualité des cours d'eau. In Etat de santé des écosystèmes aquatiques - Les variables biologiques comme indicateurs. Chartier-Touzé N., Galvin Y., Lévêque C. \& Souchon Y. (Eds.) Actes du séminaire national Hydrosystèmes, Paris, 2-3 Nov. 1994. Ed. CEMAGREF, Paris : 195-214.

Haury J., Thiébaut G. \& Muller S. 1995. — Les associations rhéophiles des rivières acides du Massif armoricain, de Lozère et des Vosges du Nord, dans un contexte Ouest-Européen. Colloq. Phytosociol. $23: 145-168$.

Holmes N.T.H. 1983. - Typing British rivers according to their flora. Focus on nature conservancy 4, Nature Conservancy Council, Huntingdon, U.K. : 194 p.

Kohler A., 1975. - Submerse Makrophyten und ihre Gesellschaften als Indikatoren der Gewässerbelastung. Beitr. Naturk. Forsch. Südwestdtschl. 34 : 149-159.

Robach F., Thiébaut G., Trémolières M. \& Muller S. 1996. - - A reference system for continental running waters : plant communities as bioindicators of increasing eutrophication in alkaline and acidic waters in the North Eastern France. Hydrobiologia, 140 : 6776.

Thiébaut G. \& Muller S. 1995. - Nouvelles données relatives à la séquence de bioindication de l'eutrophisation dans les cours d'eau faiblement minéralisés des Vosges du Nord. Acta bot. Gallica, 142 (6) : 627-638.

Thoen D., Roussel L. \& Nicolas J. 1996. — Etude des groupements de macrophytes vasculaires aquatiques de la Semois en rapport avec la qualité globale des eaux et du milieu. Ecologie, 27 (4) : 223-232.

Trémolières M., Carbiener R., Ortscheit A. \& Klein J.-P. 1994. Changes in aquatic vegetation in Rhine floodplain streams in Alsace in relation to disturbance. J. Veg. Sc., $5: 169-178$.

Université de Metz, Lab. Ecologie (coord.) 1990. - Etude des végétaux fixés en relation avec la qualité du milieu. Rapp. contr. Etude Interagence Rhin-Meuse, Rozerieulles, 3 vol. : 94 p., 64 p., $17 \mathrm{p}$.

Verneaux J. 1973. - Cours d'eau de Franche-Comté (Massif du Jura). Recherches écologiques sur le réseau hydrographique du Doubs. Thèse Doctorat, Faculté des Sciences et Techniques, Besançon : $257 \mathrm{p}$. 